\title{
Instability of standing waves for a system of nonlinear Schrödinger equations in a degenerate case
}

\author{
Shotaro Kawahara and Masahito Ohta \\ Department of Mathematics, Tokyo University of Science, \\ 1-3 Kagurazaka, Shinjuku-ku, Tokyo 162-8601, Japan
}

\begin{abstract}
We study a system of nonlinear Schrödinger equations with cubic interactions in one space dimension. The orbital stability and instability of semitrivial standing wave solutions are studied for both non-degenerate and degenerate cases.
\end{abstract}

Dedicated to Professor Nakao Hayashi on the occasion of his sixtieth birthday

\section{Introduction}

In this paper, we study the orbital stability and instability of standing wave solutions for the following system of nonlinear Schrödinger equations with cubic interactions in one space dimension:

$$
\left\{\begin{array}{l}
i \partial_{t} u_{1}=-\partial_{x}^{2} u_{1}-\kappa_{1}\left|u_{1}\right|^{2} u_{1}-\gamma u_{2}^{2} \overline{u_{1}} \\
i \partial_{t} u_{2}=-\partial_{x}^{2} u_{2}-\kappa_{2}\left|u_{2}\right|^{2} u_{2}-\gamma u_{1}^{2} \overline{u_{2}}
\end{array}\right.
$$

where $u_{1}$ and $u_{2}$ are complex-valued functions of $(t, x) \in \mathbb{R} \times \mathbb{R}$, and $\kappa_{1}$, $\kappa_{2}$ and $\gamma$ are positive constants. The system (11) appears in various areas of physics such as nonlinear optics, Bose-Einstein condensates, and so on (see, e.g., [1, 8, 9, 14]).

By the standard theory (see, e.g., [2, Chapter 4]), the Cauchy problem for (1) is globally well-posed in the energy space $H^{1}(\mathbb{R}, \mathbb{C})^{2}$, and the energy $E$ and the charge $Q$ are conserved, where

$$
\begin{aligned}
& E(\vec{u})=\sum_{j=1}^{2}\left(\frac{1}{2}\left\|\partial_{x} u_{j}\right\|_{L^{2}}^{2}-\frac{\kappa_{j}}{4}\left\|u_{j}\right\|_{L^{4}}^{4}\right)-\frac{\gamma}{2} \operatorname{Re} \int_{\mathbb{R}} u_{1}^{2}{\overline{u_{2}}}^{2} d x, \\
& Q(\vec{u})=\frac{1}{2} \sum_{j=1}^{2}\left\|u_{j}\right\|_{L^{2}}^{2}
\end{aligned}
$$


for $\vec{u}:=\left(u_{1}, u_{2}\right) \in H^{1}(\mathbb{R}, \mathbb{C})^{2}$. Note that (1) is written in a Hamiltonian form $i \partial_{t} \vec{u}=E^{\prime}(\vec{u})$, and the conservation of charge follows from the invariance of $E$ under gauge transform

$$
E\left(e^{i \theta} \vec{u}\right)=E\left(e^{i \theta} u_{1}, e^{i \theta} u_{2}\right)=E(\vec{u})
$$

for $\theta \in \mathbb{R}$ and $\vec{u} \in H^{1}(\mathbb{R}, \mathbb{C})^{2}$.

We study the orbital stability and instability of semitrivial standing wave solutions $e^{i \omega t} \vec{\phi}_{\omega}(x)$ for (1), where $\omega>0$ is a constant,

$$
\vec{\phi}_{\omega}(x):=\left(\frac{1}{\sqrt{\kappa_{1}}} \varphi_{\omega}(x), 0\right),
$$

and $\varphi_{\omega}(x)=\sqrt{2 \omega} \operatorname{sech}(\sqrt{\omega} x)$ is a positive and even solution of

$$
-\partial_{x}^{2} \varphi+\omega \varphi-\varphi^{3}=0, \quad x \in \mathbb{R} .
$$

We are mainly interested in the instability of $e^{i \omega t} \vec{\phi}_{\omega}(x)$ rather than the stability, and we assume the even symmetry for simplicity. We denote the set of even functions in $H^{1}(\mathbb{R})$ by $H_{\text {even }}^{1}(\mathbb{R})$, and define $X=H_{\text {even }}^{1}(\mathbb{R}, \mathbb{C})^{2}$. Note that $\varphi_{\omega} \in H_{\text {even }}^{1}(\mathbb{R})$ and $\vec{\phi}_{\omega} \in X$. Moreover, by the even symmetry of (11) and the uniqueness of solutions to the Cauchy problem for (11), if $\vec{u}_{0} \in X$, then the solution $\vec{u}(t)$ of (1) with $\vec{u}(0)=\vec{u}_{0}$ satisfies $\vec{u} \in C(\mathbb{R}, X)$.

Definition 1. We say that the standing wave solution $e^{i \omega t} \vec{\phi}_{\omega}$ of (11) is stable if for any $\varepsilon>0$ there exists $\delta>0$ with the following property. If $u_{0} \in X$ satisfies $\left\|\vec{u}_{0}-\vec{\phi}_{\omega}\right\|_{X}<\delta$, then the solution $\vec{u}(t)$ of (11) with $\vec{u}(0)=\vec{u}_{0}$ satisfies

$$
\inf _{\theta \in \mathbb{R}}\left\|\vec{u}(t)-e^{i \theta} \vec{\phi}_{\omega}\right\|_{X}<\varepsilon
$$

for all $t \in \mathbb{R}$. Otherwise, $e^{i \omega t} \vec{\phi}_{\omega}$ is called unstable.

We now state our main results in this paper.

Theorem 1. Let $\kappa_{1}, \kappa_{2}, \gamma$ and $\omega$ be positive constants. Then, the semitrivial standing wave solution $e^{i \omega t} \vec{\phi}_{\omega}(x)$ of (1) is stable if $\gamma<\kappa_{1}$, and unstable if $\gamma>\kappa_{1}$.

Theorem 2. Let $\kappa_{1}, \kappa_{2}, \gamma$ and $\omega$ be positive constants, and let $\gamma=\kappa_{1}$. Then, the semitrivial standing wave solution $e^{i \omega t} \vec{\phi}_{\omega}(x)$ of (11) is stable if $\kappa_{2}<\kappa_{1}$, and unstable if $\kappa_{2}>\kappa_{1}$. 
Remark 1. By symmetry, similar results to Theorems 1 and 2 also hold for semitrivial standing wave solutions of the form

$$
e^{i \omega t}\left(0, \frac{1}{\sqrt{\kappa_{2}}} \varphi_{\omega}(x)\right) \text {. }
$$

Remark 2. For the case $\gamma=\kappa_{1}=\kappa_{2}$, the system (1) has an additional symmetry

$$
\left(\begin{array}{l}
u_{1} \\
u_{2}
\end{array}\right) \mapsto R(\chi)\left(\begin{array}{l}
u_{1} \\
u_{2}
\end{array}\right), \quad R(\chi):=\left(\begin{array}{cc}
\cos \chi & -\sin \chi \\
\sin \chi & \cos \chi
\end{array}\right)
$$

for $\chi \in \mathbb{R}$. By this symmetry, in the same way as in the proof of Theorem 1 , we can prove that $e^{i \omega t} \vec{\phi}_{\omega}(x)$ is stable in the following weaker sense.

For any $\varepsilon>0$ there exists $\delta>0$ with the following property. If $u_{0} \in X$ satisfies $\left\|\vec{u}_{0}-\vec{\phi}_{\omega}\right\|_{X}<\delta$, then the solution $\vec{u}(t)$ of (1) with $\vec{u}(0)=\vec{u}_{0}$ satisfies $\inf _{\theta, \chi \in \mathbb{R}}\left\|\vec{u}(t)-e^{i \theta} R(\chi) \vec{\phi}_{\omega}\right\|_{X}<\varepsilon$ for all $t \in \mathbb{R}$.

However, we do not know whether $e^{i \omega t} \vec{\phi}_{\omega}(x)$ is stable or not in the sense of Definition 1 for the case $\gamma=\kappa_{1}=\kappa_{2}$.

Remark 3. The standing waves $e^{i \omega t} \vec{\phi}_{\omega}(x)$ are also solutions of the following system

$$
\left\{\begin{array}{l}
i \partial_{t} u_{1}=-\partial_{x}^{2} u_{1}-\kappa_{1}\left|u_{1}\right|^{2} u_{1}-\gamma\left|u_{2}\right|^{2} u_{1} \\
i \partial_{t} u_{2}=-\partial_{x}^{2} u_{2}-\kappa_{2}\left|u_{2}\right|^{2} u_{2}-\gamma\left|u_{1}\right|^{2} u_{2}
\end{array}\right.
$$

It is known that for any positive constants $\kappa_{1}, \kappa_{2}, \gamma$ and $\omega$, the standing wave solution $e^{i \omega t} \vec{\phi}_{\omega}(x)$ is stable for (3) (see [12, 11]).

Remark 4. For related results on systems of nonlinear Schödinger equations with quadratic interactions, see [5, 77]. While, for related studies on degenerate cases, see [10, 17].

The rest of the paper is organized as follows. In section 2, we consider the non-degenerate case $\gamma \neq \kappa_{1}$. The stability part of Theorem 1 is proved by the standard argument based on [6, 16]. The degenerate case $\gamma=\kappa_{1}$ is studied in section 3. The instability part of Theorem 2 is proved by using similar arguments to those in [5, 13].

\section{Proof of Theorem 1}

We regard $L^{2}(\mathbb{R}, \mathbb{C})$ as a real Hilbert space with the inner product

$$
(u, v)_{L^{2}}=\operatorname{Re} \int_{\mathbb{R}} u(x) \overline{v(x)} d x
$$


and define the inner products of real Hilbert spaces $H=L_{\text {even }}^{2}(\mathbb{R}, \mathbb{C})^{2}$ and $X=H_{\text {even }}^{1}(\mathbb{R}, \mathbb{C})^{2}$ by

$$
(\vec{u}, \vec{v})_{H}=\left(u_{1}, v_{1}\right)_{L^{2}}+\left(u_{2}, v_{2}\right)_{L^{2}}, \quad(\vec{u}, \vec{v})_{X}=(\vec{u}, \vec{v})_{H}+\left(\partial_{x} \vec{u}, \partial_{x} \vec{v}\right)_{H} .
$$

For $\omega>0$, we define $S_{\omega}(\vec{v})=E(\vec{v})+\omega Q(\vec{v})$ for $\vec{v} \in X$. Then, we have $S_{\omega}^{\prime}\left(\vec{\phi}_{\omega}\right)=0$. Moreover, for $a \in \mathbb{R}$, we define $L_{a}$ by

$$
L_{a} u=-\partial_{x}^{2} u+\omega u-a \varphi_{\omega}(x)^{2} u
$$

for $u \in H_{\text {even }}^{1}(\mathbb{R}, \mathbb{R})$. Then, for $\vec{v}=\left(v_{1}, v_{2}\right) \in X$, we have

$$
\begin{aligned}
\left\langle S_{\omega}^{\prime \prime}\left(\vec{\phi}_{\omega}\right) \vec{v}, \vec{v}\right\rangle= & \left\langle L_{3} \operatorname{Re} v_{1}, \operatorname{Re} v_{1}\right\rangle+\left\langle L_{1} \operatorname{Im} v_{1}, \operatorname{Im} v_{1}\right\rangle \\
& +\left\langle L_{\gamma / \kappa_{1}} \operatorname{Re} v_{2}, \operatorname{Re} v_{2}\right\rangle+\left\langle L_{-\gamma / \kappa_{1}} \operatorname{Im} v_{2}, \operatorname{Im} v_{2}\right\rangle .
\end{aligned}
$$

We recall some known results on $L_{a}$ (see [15]).

Lemma 1. (1) If $1 \leq a \leq 3$, then there exists $C>0$ such that $\left\langle L_{a} v, v\right\rangle \geq$ $C\|v\|_{H^{1}}^{2}$ for all $v \in H_{\text {even }}^{1}(\mathbb{R}, \mathbb{R})$ satisfying $\left(v, \varphi_{\omega}\right)_{L^{2}}=0$.

(2) If $a<1$, then there exists $C>0$ such that $\left\langle L_{a} v, v\right\rangle \geq C\|v\|_{H^{1}}^{2}$ for all $v \in H_{\text {even }}^{1}(\mathbb{R}, \mathbb{R})$.

(3) $L_{1} \varphi_{\omega}=0$. If $a>1$, then $\left\langle L_{a} \varphi_{\omega}, \varphi_{\omega}\right\rangle<0$.

To prove the stability part of Theorem 1, we use the following proposition (see [6, 16]).

Proposition 2. Assume that there exists a constant $C>0$ such that $\left\langle S_{\omega}^{\prime \prime}\left(\vec{\phi}_{\omega}\right) \vec{w}, \vec{w}\right\rangle \geq C\|\vec{w}\|_{X}^{2}$ for all $\vec{w} \in X$ satisfying

$$
\left(\vec{w}, \vec{\phi}_{\omega}\right)_{H}=\left(\vec{w}, i \vec{\phi}_{\omega}\right)_{H}=0 .
$$

Then, the standing wave solution $e^{i \omega t} \vec{\phi}_{\omega}$ of (1) is stable.

Proof of Theorem 1 (Stability part). Assume that $\gamma<\kappa_{1}$.

By Lemma 1 (1), there exists $C_{1}>0$ such that

$$
\left\langle L_{3} \operatorname{Re} w_{1}, \operatorname{Re} w_{1}\right\rangle+\left\langle L_{1} \operatorname{Im} w_{1}, \operatorname{Im} w_{1}\right\rangle \geq C_{1}\left\|w_{1}\right\|_{H^{1}}^{2}
$$

for all $w_{1} \in H^{1}(\mathbb{R}, \mathbb{C})$ satisfying

$$
\left(\operatorname{Re} w_{1}, \varphi_{\omega}\right)_{L^{2}}=\left(\operatorname{Im} w_{1}, \varphi_{\omega}\right)_{L^{2}}=0 .
$$

Note that since $\vec{\phi}_{\omega}$ has the form (2), the condition (6) is equivalent to (5). Moreover, by the assumption $0<\gamma<\kappa_{1}$, we have $-\gamma / \kappa_{1}<\gamma / \kappa_{1}<1$. Thus, by Lemma 1 (2), there exists $C_{2}>0$ such that

$$
\left\langle L_{\gamma / \kappa_{1}} \operatorname{Re} w_{2}, \operatorname{Re} w_{2}\right\rangle+\left\langle L_{-\gamma / \kappa_{1}} \operatorname{Im} w_{2}, \operatorname{Im} w_{2}\right\rangle \geq C_{2}\left\|w_{2}\right\|_{H^{1}}^{2}
$$


for all $w_{2} \in H_{\text {even }}^{1}(\mathbb{R}, \mathbb{C})$.

Thus, putting $C_{3}=\min \left\{C_{1}, C_{2}\right\}$, we have $\left\langle S_{\omega}^{\prime \prime}\left(\vec{\phi}_{\omega}\right) \vec{w}, \vec{w}\right\rangle \geq C_{3}\|\vec{w}\|_{X}^{2}$ for all $\vec{w} \in X$ satisfying (5). Hence, the stability part of Theorem 1 follows from Proposition 2 .

Next, we consider the instability part of Theorem 1. The instability of $e^{i \omega t} \vec{\phi}_{\omega}$ can be proved for all $\gamma \in\left(\kappa_{1}, \infty\right)$ in the same way as in [3, 4] using the linear instability argument. On the other hand, by the Lyapunov function method, the instability of $e^{i \omega t} \vec{\phi}_{\omega}$ is proved for a restricted case $\gamma \in\left(\kappa_{1}, 3 \kappa_{1}\right]$. Since our main interest in this paper is to consider the borderline case $\gamma=\kappa_{1}$ in Theorem 2, and since the instability result in Theorem 2 is proved by the Lyapunov function method but not by the linear instability argument, we here give the proof of instability for the case $\gamma \in\left(\kappa_{1}, 3 \kappa_{1}\right]$. To prove the instability of $e^{i \omega t} \vec{\phi}_{\omega}$ for this case, we use the following proposition (see [13]).

Proposition 3. Assume that there exist $\vec{\psi} \in X$ and a constant $C>0$ such that

$$
\left(\vec{\psi}, \vec{\phi}_{\omega}\right)_{H}=\left(\vec{\psi}, i \vec{\phi}_{\omega}\right)_{H}=0, \quad\left\langle S_{\omega}^{\prime \prime}\left(\vec{\phi}_{\omega}\right) \vec{\psi}, \vec{\psi}\right\rangle<0,
$$

and $\left\langle S_{\omega}^{\prime \prime}\left(\vec{\phi}_{\omega}\right) \vec{w}, \vec{w}\right\rangle \geq C\|\vec{w}\|_{X}^{2}$ for all $\vec{w} \in X$ satisfying

$$
\left(\vec{w}, \vec{\phi}_{\omega}\right)_{H}=\left(\vec{w}, i \vec{\phi}_{\omega}\right)_{H}=(\vec{w}, \vec{\psi})_{H}=0 .
$$

Then, the standing wave solution $e^{i \omega t} \vec{\phi}_{\omega}$ of (11) is unstable.

Proof of Theorem 1 (Instability part for the case $\kappa_{1}<\gamma \leq 3 \kappa_{1}$ ).

Assume that $\gamma \in\left(\kappa_{1}, 3 \kappa_{1}\right]$. We take

$$
\vec{\psi}_{\omega}=\left(0, \frac{1}{\sqrt{\kappa_{1}}} \varphi_{\omega}\right) .
$$

Then, $\vec{\psi}_{\omega} \in X$ and $\left(\vec{\psi}_{\omega}, \vec{\phi}_{\omega}\right)_{H}=\left(\vec{\psi}_{\omega}, i \vec{\phi}_{\omega}\right)_{H}=0$.

Since $1<\gamma / \kappa_{1} \leq 3$, by Lemma 1 (3), we have

$$
\left\langle S_{\omega}^{\prime \prime}\left(\vec{\phi}_{\omega}\right) \vec{\psi}_{\omega}, \vec{\psi}_{\omega}\right\rangle=\frac{1}{\kappa_{1}}\left\langle L_{\gamma / \kappa_{1}} \varphi_{\omega}, \varphi_{\omega}\right\rangle<0 .
$$

Moreover, since the condition (77) is equivalent to

$$
\left(\operatorname{Re} w_{1}, \varphi_{\omega}\right)_{L^{2}}=\left(\operatorname{Im} w_{1}, \varphi_{\omega}\right)_{L^{2}}=\left(\operatorname{Re} w_{2}, \varphi_{\omega}\right)_{L^{2}}=0,
$$

by Lemma 1 (1) and (2), there exists a constant $C>0$ such that $\left\langle S_{\omega}^{\prime \prime}\left(\vec{\phi}_{\omega}\right) \vec{w}, \vec{w}\right\rangle \geq C\|\vec{w}\|_{X}^{2}$ for all $\vec{w} \in X$ satisfying (77).

Hence, the instability of $e^{i \omega t} \vec{\phi}_{\omega}$ follows from Proposition 3 . 


\section{Proof of Theorem 2}

In this section, we consider the case $\gamma=\kappa_{1}$. By (4), we have

$$
\begin{aligned}
\left\langle S_{\omega}^{\prime \prime}\left(\vec{\phi}_{\omega}\right) \vec{v}, \vec{v}\right\rangle= & \left\langle L_{3} \operatorname{Re} v_{1}, \operatorname{Re} v_{1}\right\rangle+\left\langle L_{1} \operatorname{Im} v_{1}, \operatorname{Im} v_{1}\right\rangle \\
& +\left\langle L_{1} \operatorname{Re} v_{2}, \operatorname{Re} v_{2}\right\rangle+\left\langle L_{-1} \operatorname{Im} v_{2}, \operatorname{Im} v_{2}\right\rangle
\end{aligned}
$$

for $\vec{v}=\left(v_{1}, v_{2}\right) \in X$. Recall that

$$
\vec{\phi}_{\omega}=\left(\frac{1}{\sqrt{\kappa_{1}}} \varphi_{\omega}, 0\right), \quad \vec{\psi}_{\omega}=\left(0, \frac{1}{\sqrt{\kappa_{1}}} \varphi_{\omega}\right) .
$$

Then, we have

$$
\begin{aligned}
& \left\|\vec{\psi}_{\omega}\right\|_{H}=\left\|\vec{\phi}_{\omega}\right\|_{H}, \quad\left(\vec{\psi}_{\omega}, \vec{\phi}_{\omega}\right)_{H}=\left(\vec{\psi}_{\omega}, i \vec{\phi}_{\omega}\right)_{H}=0 \\
& S_{\omega}^{\prime \prime}\left(\vec{\phi}_{\omega}\right) \vec{\psi}_{\omega}=\left(0, \frac{1}{\sqrt{\kappa_{1}}} L_{1} \varphi_{\omega}\right)=(0,0), \\
& S_{\omega}^{\prime \prime}\left(\vec{\phi}_{\omega}\right) \vec{\phi}_{\omega}=S_{\omega}^{(3)}\left(\vec{\phi}_{\omega}\right)\left(\vec{\psi}_{\omega}, \vec{\psi}_{\omega}\right)=\left(-\frac{2}{\sqrt{\kappa_{1}}} \varphi_{\omega}^{3}, 0\right) .
\end{aligned}
$$

In particular, we have

$$
\left\langle S_{\omega}^{(3)}\left(\vec{\phi}_{\omega}\right)\left(\vec{\psi}_{\omega}, \vec{\psi}_{\omega}\right), \vec{\psi}_{\omega}\right\rangle=0
$$

Moreover, we put

$$
\begin{aligned}
\nu_{1} & :=\left\langle S_{\omega}^{(4)}\left(\vec{\phi}_{\omega}\right)\left(\vec{\psi}_{\omega}, \vec{\psi}_{\omega}, \vec{\psi}_{\omega}\right), \vec{\psi}_{\omega}\right\rangle, \\
\nu_{0} & :=\frac{1}{8}\left\langle S_{\omega}^{\prime \prime}\left(\vec{\phi}_{\omega}\right) \vec{\phi}_{\omega}, \vec{\phi}_{\omega}\right\rangle-\frac{1}{4}\left\langle S_{\omega}^{(3)}\left(\vec{\phi}_{\omega}\right)\left(\vec{\psi}_{\omega}, \vec{\psi}_{\omega}\right), \vec{\phi}_{\omega}\right\rangle+\frac{1}{4 !} \nu_{1} .
\end{aligned}
$$

Then, by simple computations, we have

$$
\nu_{1}=-\frac{6 \kappa_{2}}{\kappa_{1}^{2}}\left\|\varphi_{\omega}\right\|_{L^{4}}^{4}, \quad \nu_{0}=\frac{\kappa_{1}-\kappa_{2}}{4 \kappa_{1}^{2}}\left\|\varphi_{\omega}\right\|_{L^{4}}^{4}
$$

As we will see below, the sign of $\nu_{0}$ determines the stability and instability of $e^{i \omega t} \vec{\phi}_{\omega}$ for the borderline case $\gamma=\kappa_{1}$.

The following lemma plays an important role in the proof of Theorem 2 for both stability and instability results.

Lemma 4. There exists a constant $k_{0}>0$ such that

$$
\left\langle S_{\omega}^{\prime \prime}\left(\phi_{\omega}\right) \vec{w}, \vec{w}\right\rangle \geq k_{0}\|\vec{w}\|_{X}^{2}
$$

for all $\vec{w} \in W$, where

$$
W=\left\{\vec{w} \in X:\left(\vec{w}, \vec{\phi}_{\omega}\right)_{H}=\left(\vec{w}, i \vec{\phi}_{\omega}\right)_{H}=\left(\vec{w}, \vec{\psi}_{\omega}\right)_{H}=0\right\} .
$$


Proof. Since $\vec{w} \in W$ satisfies

$$
\left(\operatorname{Re} w_{1}, \varphi_{\omega}\right)_{L^{2}}=\left(\operatorname{Im} w_{1}, \varphi_{\omega}\right)_{L^{2}}=\left(\operatorname{Re} w_{2}, \varphi_{\omega}\right)_{L^{2}}=0,
$$

the conclusion follows from (8) and Lemma 1,

Lemma 5. For $\lambda \in \mathbb{R}$,

$$
S_{\omega}\left(\vec{\phi}_{\omega}+\lambda \vec{\psi}_{\omega}\right)=S_{\omega}\left(\vec{\phi}_{\omega}\right)+\frac{\nu_{1}}{4 !} \lambda^{4}, \quad\left\langle S_{\omega}^{\prime}\left(\vec{\phi}_{\omega}+\lambda \vec{\psi}_{\omega}\right), \vec{\psi}_{\omega}\right\rangle=\frac{\nu_{1}}{3 !} \lambda^{3} .
$$

Proof. By Taylor's expansion, we have

$$
\begin{aligned}
S_{\omega}\left(\vec{\phi}_{\omega}\right. & \left.+\lambda \vec{\psi}_{\omega}\right)=S_{\omega}\left(\vec{\phi}_{\omega}\right)+\lambda\left\langle S_{\omega}^{\prime}\left(\vec{\phi}_{\omega}\right), \vec{\psi}_{\omega}\right\rangle+\frac{\lambda^{2}}{2}\left\langle S_{\omega}^{\prime \prime}\left(\vec{\phi}_{\omega}\right) \vec{\psi}_{\omega}, \vec{\psi}_{\omega}\right\rangle \\
& +\frac{\lambda^{3}}{3 !}\left\langle S_{\omega}^{(3)}\left(\vec{\phi}_{\omega}\right)\left(\vec{\psi}_{\omega}, \vec{\psi}_{\omega}\right), \vec{\psi}_{\omega}\right\rangle+\frac{\lambda^{4}}{4 !}\left\langle S_{\omega}^{(4)}\left(\vec{\phi}_{\omega}\right)\left(\vec{\psi}_{\omega}, \vec{\psi}_{\omega}, \vec{\psi}_{\omega}\right), \vec{\psi}_{\omega}\right\rangle .
\end{aligned}
$$

Since $S_{\omega}^{\prime}\left(\vec{\phi}_{\omega}\right)=S_{\omega}^{\prime \prime}\left(\vec{\phi}_{\omega}\right) \vec{\psi}_{\omega}=0$ and $\left\langle S_{\omega}^{(3)}\left(\vec{\phi}_{\omega}\right)\left(\vec{\psi}_{\omega}, \vec{\psi}_{\omega}\right), \vec{\psi}_{\omega}\right\rangle=0$, we have

$$
S_{\omega}\left(\vec{\phi}_{\omega}+\lambda \vec{\psi}_{\omega}\right)=S_{\omega}\left(\vec{\phi}_{\omega}\right)+\frac{\nu_{1}}{4 !} \lambda^{4} .
$$

Moreover, by differentiating this identity with respect to $\lambda$, we have the second identity.

Lemma 6. For $\lambda \in \mathbb{R}$,

$$
S_{\omega}^{\prime}\left(\vec{\phi}_{\omega}+\lambda \vec{\psi}_{\omega}\right)=\frac{\lambda^{2}}{2} S_{\omega}^{(3)}\left(\vec{\phi}_{\omega}\right)\left(\vec{\psi}_{\omega}, \vec{\psi}_{\omega}\right)+o\left(\lambda^{2}\right) .
$$

Proof. Since $S_{\omega}^{\prime}\left(\vec{\phi}_{\omega}\right)=S_{\omega}^{\prime \prime}\left(\vec{\phi}_{\omega}\right) \vec{\psi}_{\omega}=0$, we have

$$
\begin{aligned}
& S_{\omega}^{\prime}\left(\vec{\phi}_{\omega}+\lambda \vec{\psi}_{\omega}\right) \\
& =S_{\omega}^{\prime}\left(\vec{\phi}_{\omega}\right)+\lambda S_{\omega}^{\prime \prime}\left(\vec{\phi}_{\omega}\right) \vec{\psi}_{\omega}+\frac{\lambda^{2}}{2} S_{\omega}^{(3)}\left(\vec{\phi}_{\omega}\right)\left(\vec{\psi}_{\omega}, \vec{\psi}_{\omega}\right)+o\left(\lambda^{2}\right) \\
& =\frac{\lambda^{2}}{2} S_{\omega}^{(3)}\left(\vec{\phi}_{\omega}\right)\left(\vec{\psi}_{\omega}, \vec{\psi}_{\omega}\right)+o\left(\lambda^{2}\right) .
\end{aligned}
$$

This completes the proof.

Lemma 7. For $\lambda \in \mathbb{R}$ and $\vec{z} \in X$,

$$
\begin{aligned}
& S_{\omega}\left(\vec{\phi}_{\omega}+\lambda \vec{\psi}_{\omega}+\vec{z}\right)-S_{\omega}\left(\vec{\phi}_{\omega}\right) \\
& =\frac{\nu_{1}}{4 !} \lambda^{4}+\frac{\lambda^{2}}{2}\left\langle S_{\omega}^{(3)}\left(\vec{\phi}_{\omega}\right)\left(\vec{\psi}_{\omega}, \vec{\psi}_{\omega}\right), \vec{z}\right\rangle+\frac{1}{2}\left\langle S_{\omega}^{\prime \prime}\left(\vec{\phi}_{\omega}\right) \vec{z}, \vec{z}\right\rangle \\
& \quad+o\left(\lambda^{4}+\|\vec{z}\|_{X}^{2}\right) .
\end{aligned}
$$


Proof. By Taylor's expansion, we have

$$
\begin{aligned}
S_{\omega}\left(\vec{\phi}_{\omega}\right. & \left.+\lambda \vec{\psi}_{\omega}+\vec{z}\right)=S_{\omega}\left(\vec{\phi}_{\omega}+\lambda \vec{\psi}_{\omega}\right) \\
& +\left\langle S_{\omega}^{\prime}\left(\vec{\phi}_{\omega}+\lambda \vec{\psi}_{\omega}\right), \vec{z}\right\rangle+\frac{1}{2}\left\langle S_{\omega}^{\prime \prime}\left(\vec{\phi}_{\omega}+\lambda \vec{\psi}_{\omega}\right) \vec{z}, \vec{z}\right\rangle+o\left(\|\vec{z}\|_{X}^{2}\right) .
\end{aligned}
$$

Here, by Lemma 5, we have $S_{\omega}\left(\vec{\phi}_{\omega}+\lambda \vec{\psi}_{\omega}\right)=S_{\omega}\left(\vec{\phi}_{\omega}\right)+\frac{\nu_{1}}{4 !} \lambda^{4}$.

Next, it follows from Lemma 6 that

$$
\left\langle S_{\omega}^{\prime}\left(\vec{\phi}_{\omega}+\lambda \vec{\psi}_{\omega}\right), \vec{z}\right\rangle=\frac{\lambda^{2}}{2}\left\langle S_{\omega}^{(3)}\left(\vec{\phi}_{\omega}\right)\left(\vec{\psi}_{\omega}, \vec{\psi}_{\omega}\right), \vec{z}\right\rangle+o\left(\lambda^{4}+\|\vec{z}\|_{X}^{2}\right) .
$$

Moreover, we have

$$
\left\langle S_{\omega}^{\prime \prime}\left(\vec{\phi}_{\omega}+\lambda \vec{\psi}_{\omega}\right) \vec{z}, \vec{z}\right\rangle=\left\langle S_{\omega}^{\prime \prime}\left(\vec{\phi}_{\omega}\right) \vec{z}, \vec{z}\right\rangle+O\left(\lambda\|\vec{z}\|_{X}^{2}\right) .
$$

Thus, we have the desired estimate.

Lemma 8. Let $\vec{v}=\lambda \vec{\psi}_{\omega}+\mu \vec{\phi}_{\omega}+\vec{w}$ with $\lambda, \mu \in \mathbb{R}$ and $\vec{w} \in W$. Assume that $\left\|\vec{\phi}_{\omega}+\vec{v}\right\|_{H}^{2}=\left\|\vec{\phi}_{\omega}\right\|_{H}^{2}$. Then,

$$
\mu=-\frac{\lambda^{2}}{2}+O\left(\mu^{2}+\|\vec{w}\|_{X}^{2}\right)
$$

Proof. Since $\vec{\psi}_{\omega}, \vec{\phi}_{\omega}$ and $\vec{w}$ are orthogonal to each other in $H$, we have

$$
\left\|\vec{\phi}_{\omega}\right\|_{H}^{2}=\left\|\vec{\phi}_{\omega}+\vec{v}\right\|_{H}^{2}=\lambda^{2}\left\|\vec{\psi}_{\omega}\right\|_{H}^{2}+(1+\mu)^{2}\left\|\vec{\phi}_{\omega}\right\|_{H}^{2}+\|\vec{w}\|_{H}^{2} .
$$

Moreover, since $\left\|\vec{\psi}_{\omega}\right\|_{H}=\left\|\vec{\phi}_{\omega}\right\|_{H}$, we have

$$
\mu=-\frac{\lambda^{2}}{2}+\frac{1}{2}\left(\mu^{2}+\frac{\|\vec{w}\|_{H}^{2}}{\left\|\vec{\phi}_{\omega}\right\|_{H}^{2}}\right),
$$

which implies the desired result.

Lemma 9. Let $\vec{v}=\lambda \vec{\psi}_{\omega}+\mu \vec{\phi}_{\omega}+\vec{w}$ with $\lambda, \mu \in \mathbb{R}$ and $\vec{w} \in W$. Assume that $\left\|\vec{\phi}_{\omega}+\vec{v}\right\|_{H}^{2}=\left\|\vec{\phi}_{\omega}\right\|_{H}^{2}$. Then,

$$
E\left(\vec{\phi}_{\omega}+\vec{v}\right)-E\left(\vec{\phi}_{\omega}\right)=\nu_{0} \lambda^{4}+\frac{1}{2}\left\langle S_{\omega}^{\prime \prime}\left(\vec{\phi}_{\omega}\right) \vec{w}, \vec{w}\right\rangle+o\left(\lambda^{4}+\|\vec{w}\|_{X}^{2}\right) .
$$


Proof. By Lemmas 7 and 8, we have

$$
\begin{aligned}
E & \left(\vec{\phi}_{\omega}+\vec{v}\right)-E\left(\vec{\phi}_{\omega}\right)=S_{\omega}\left(\vec{\phi}_{\omega}+\vec{v}\right)-S_{\omega}\left(\vec{\phi}_{\omega}\right) \\
= & S_{\omega}\left(\vec{\phi}_{\omega}+\lambda \vec{\psi}_{\omega}+\mu \vec{\phi}_{\omega}+\vec{w}\right)-S_{\omega}\left(\vec{\phi}_{\omega}\right) \\
= & \frac{\nu_{1}}{4 !} \lambda^{4}+\frac{\lambda^{2}}{2}\left\langle S_{\omega}^{(3)}\left(\vec{\phi}_{\omega}\right)\left(\vec{\psi}_{\omega}, \vec{\psi}_{\omega}\right), \mu \vec{\phi}_{\omega}+\vec{w}\right\rangle \\
& +\frac{1}{2}\left\langle S_{\omega}^{\prime \prime}\left(\vec{\phi}_{\omega}\right)\left(\mu \vec{\phi}_{\omega}+\vec{w}\right), \mu \vec{\phi}_{\omega}+\vec{w}\right\rangle+o\left(\lambda^{4}+\left\|\mu \vec{\phi}_{\omega}+\vec{w}\right\|_{X}^{2}\right) \\
= & \nu_{0} \lambda^{4}+\frac{\lambda^{2}}{2}\left\langle S_{\omega}^{(3)}\left(\vec{\phi}_{\omega}\right)\left(\vec{\psi}_{\omega}, \vec{\psi}_{\omega}\right), \vec{w}\right\rangle-\frac{\lambda^{2}}{2}\left\langle S_{\omega}^{\prime \prime}\left(\vec{\phi}_{\omega}\right) \vec{\phi}_{\omega}, \vec{w}\right\rangle \\
& +\frac{1}{2}\left\langle S_{\omega}^{\prime \prime}\left(\vec{\phi}_{\omega}\right) \vec{w}, \vec{w}\right\rangle+o\left(\lambda^{4}+\|\vec{w}\|_{X}^{2}\right) .
\end{aligned}
$$

Here, by (9), the second and the third terms in the last equation cancel each other out. This completes the proof.

To prove the stability part of Theorem 2, we use the following proposition (see [6]). For $\varepsilon>0$, we define

$$
U_{\varepsilon}\left(\vec{\phi}_{\omega}\right)=\left\{\vec{u} \in X: \inf _{\theta \in \mathbb{R}}\left\|\vec{u}-e^{i \theta} \vec{\phi}_{\omega}\right\|_{X}<\varepsilon\right\} .
$$

Proposition 10. Assume that there exist positive constants $p, C$ and $\varepsilon$ such that

$$
E(\vec{u}) \geq E\left(\vec{\phi}_{\omega}\right)+C \inf _{\theta \in \mathbb{R}}\left\|\vec{u}-e^{i \theta} \vec{\phi}_{\omega}\right\|_{X}^{p}
$$

for all $\vec{u} \in U_{\varepsilon}\left(\vec{\phi}_{\omega}\right)$ satisfying $Q(\vec{u})=Q\left(\vec{\phi}_{\omega}\right)$. Then, the standing wave solution $e^{i \omega t} \vec{\phi}_{\omega}$ of (1) is stable.

Before proving the stability part of Theorem 2, we prepare one more lemma.

Lemma 11. There exist $\varepsilon>0$ and a $C^{2}$-function $\alpha: U_{\varepsilon}\left(\vec{\phi}_{\omega}\right) \rightarrow \mathbb{R} / 2 \pi \mathbb{Z}$ such that

$$
\begin{aligned}
& \left\|\vec{u}-e^{i \alpha(\vec{u})} \vec{\phi}_{\omega}\right\|_{H} \leq\left\|\vec{u}-e^{i \theta} \vec{\phi}_{\omega}\right\|_{H}, \quad \alpha\left(e^{i \theta} \vec{u}\right)=\alpha(\vec{u})+\theta, \\
& \left(\vec{u}, e^{i \alpha(\vec{u})} i \vec{\phi}_{\omega}\right)_{H}=0, \quad i \alpha^{\prime}(\vec{u})=-\frac{e^{i \alpha(\vec{u})} \vec{\phi}_{\omega}}{\left(\vec{u}, e^{i \alpha(\vec{u})} \vec{\phi}_{\omega}\right)_{H}}
\end{aligned}
$$

for all $\vec{u} \in U_{\varepsilon}\left(\vec{\phi}_{\omega}\right)$ and $\theta \in \mathbb{R} / 2 \pi \mathbb{Z}$.

Proof. See Lemma 3.2 of [6]. 
Proof of Theorem 2 (Stability part). Assume that $\gamma=\kappa_{1}>\kappa_{2}$.

Let $\vec{u} \in U_{\varepsilon}\left(\vec{\phi}_{\omega}\right)$ with $Q(\vec{u})=Q\left(\vec{\phi}_{\omega}\right)$. Then, for $\alpha(\vec{u})$ given in Lemma 11, we have

$$
\left\|\vec{u}-e^{i \alpha(\vec{u})} \vec{\phi}_{\omega}\right\|_{X} \leq\left(1+\frac{2\left\|\vec{\phi}_{\omega}\right\|_{X}}{\left\|\vec{\phi}_{\omega}\right\|_{H}}\right) \varepsilon
$$

Indeed, for $\beta(\vec{u}) \in \mathbb{R}$ such that

$$
\left\|\vec{u}-e^{i \beta(\vec{u})} \vec{\phi}_{\omega}\right\|_{X}=\inf _{\theta \in \mathbb{R}}\left\|\vec{u}-e^{i \theta} \vec{\phi}_{\omega}\right\|_{X}<\varepsilon
$$

we have

$$
\begin{aligned}
& \left|e^{i \alpha(\vec{u})}-e^{i \beta(\vec{u})}\right|\left\|\vec{\phi}_{\omega}\right\|_{H} \leq\left\|e^{i \alpha(\vec{u})} \vec{\phi}_{\omega}-\vec{u}\right\|_{H}+\left\|\vec{u}-e^{i \beta(\vec{u})} \vec{\phi}_{\omega}\right\|_{H} \\
& \leq 2\left\|\vec{u}-e^{i \beta(\vec{u})} \vec{\phi}_{\omega}\right\|_{H} \leq 2\left\|\vec{u}-e^{i \beta(\vec{u})} \vec{\phi}_{\omega}\right\|_{X}<2 \varepsilon
\end{aligned}
$$

and $\left\|\vec{u}-e^{i \alpha(\vec{u})} \vec{\phi}_{\omega}\right\|_{X} \leq\left\|\vec{u}-e^{i \beta(\vec{u})} \vec{\phi}_{\omega}\right\|_{X}+\left|e^{i \alpha(\vec{u})}-e^{i \beta(\vec{u})}\right|\left\|\vec{\phi}_{\omega}\right\|_{X}$, which implies (12).

Let $\vec{v}=e^{-i \alpha(\vec{u})} \vec{u}-\vec{\phi}_{\omega}$. Then, we have $\left(\vec{v}, i \vec{\phi}_{\omega}\right)_{H}=0$, and we decompose $\vec{v}$ as $\vec{v}=\lambda \vec{\psi}_{\omega}+\mu \vec{\phi}_{\omega}+\vec{w}$ with $\lambda, \mu \in \mathbb{R}$ and $\vec{w} \in W$.

Since $\left\|\vec{\phi}_{\omega}+\vec{v}\right\|_{H}^{2}=\|\vec{u}\|_{H}^{2}=2 Q(\vec{u})=2 Q\left(\vec{\phi}_{\omega}\right)=\left\|\vec{\phi}_{\omega}\right\|_{H}^{2}$, it follows from Lemmas 9 and 4 that

$$
E(\vec{u})-E\left(\vec{\phi}_{\omega}\right) \geq \nu_{0} \lambda^{4}+\frac{k_{0}}{2}\|\vec{w}\|_{X}^{2}+o\left(\lambda^{4}+\|\vec{w}\|_{X}^{2}\right)
$$

Here, we note that $k_{0}$ is the positive constant given in Lemma 4, and that $\nu_{0}>0$ by (10) and the assumption $\kappa_{1}>\kappa_{2}$.

Moreover, by Lemma 8, we have

$$
\begin{aligned}
& \inf _{\theta \in \mathbb{R}}\left\|\vec{u}-e^{i \theta} \vec{\phi}_{\omega}\right\|_{X} \leq\|\vec{v}\|_{X} \leq|\lambda|\left\|\vec{\psi}_{\omega}\right\|_{X}+|\mu|\left\|\vec{\phi}_{\omega}\right\|_{X}+\|\vec{w}\|_{X} \\
& =|\lambda|\left\|\vec{\psi}_{\omega}\right\|_{X}+\|\vec{w}\|_{X}+O\left(\lambda^{2}+\|\vec{w}\|_{X}^{2}\right) .
\end{aligned}
$$

Thus, taking $\varepsilon$ smaller if necessary, we have

$$
E(\vec{u})-E\left(\vec{\phi}_{\omega}\right) \geq \frac{\nu_{0}}{2} \lambda^{4}+\frac{k_{0}}{4}\|\vec{w}\|_{X}^{2} \geq C_{1} \inf _{\theta \in \mathbb{R}}\left\|\vec{u}-e^{i \theta} \vec{\phi}_{\omega}\right\|_{X}^{4}
$$

for some $C_{1}>0$.

Hence, the stability of $e^{i \omega t} \vec{\phi}_{\omega}$ follows from Proposition 10.

In the rest of this section, we study the instability of $e^{i \omega t} \vec{\phi}_{\omega}$ for the case $\gamma=\kappa_{1}<\kappa_{2}$. We follow the argument used in [5, 13]. 
For $\vec{u} \in U_{\varepsilon}\left(\vec{\phi}_{\omega}\right)$, we define

$$
\begin{aligned}
& M(\vec{u})=e^{-i \alpha(\vec{u})} \vec{u}, \quad A(\vec{u})=\left(i M(\vec{u}), \vec{\psi}_{\omega}\right)_{H}, \\
& q(\vec{u})=e^{i \alpha(\vec{u})} \vec{\psi}_{\omega}+\left(M(\vec{u}), \vec{\psi}_{\omega}\right)_{H} i \alpha^{\prime}(\vec{u}), \\
& P(\vec{u})=\left\langle E^{\prime}(\vec{u}), q(\vec{u})\right\rangle .
\end{aligned}
$$

Then, we have the following lemmas (see [6]).

Lemma 12. For $\vec{u} \in U_{\varepsilon}\left(\vec{\phi}_{\omega}\right)$,

(1) $A\left(e^{i \theta} \vec{u}\right)=A(\vec{u}), \quad q\left(e^{i \theta} \vec{u}\right)=e^{i \theta} q(\vec{u})$ for all $\theta \in \mathbb{R}$.

(2) $\left\langle A^{\prime}(\vec{u}), \vec{w}\right\rangle=(q(\vec{u}), i \vec{w})_{H}$ for $\vec{w} \in X$.

(3) $q\left(\vec{\phi}_{\omega}\right)=\vec{\psi}_{\omega}, \quad\left\langle Q^{\prime}(\vec{u}), q(\vec{u})\right\rangle=0$.

Lemma 13. Let $I$ be an interval of $\mathbb{R}$. Let $\vec{u} \in C(I, X)$ be a solution of (1), and assume that $\vec{u}(t) \in U_{\varepsilon}\left(\vec{\phi}_{\omega}\right)$ for all $t \in I$. Then,

$$
\frac{d}{d t} A(\vec{u}(t))=P(\vec{u}(t)) \quad \text { for all } t \in I .
$$

By Lemma 12 and (11), we have

$$
\begin{aligned}
& P(\vec{u})=\left\langle S_{\omega}^{\prime}(\vec{u}), q(\vec{u})\right\rangle \\
& =\left\langle S_{\omega}^{\prime}(M(\vec{u})), \vec{\psi}_{\omega}\right\rangle-\frac{\left(M(\vec{u}), \vec{\psi}_{\omega}\right)_{H}}{\left(M(\vec{u}), \vec{\phi}_{\omega}\right)_{H}}\left\langle S_{\omega}^{\prime}(M(\vec{u})), \vec{\phi}_{\omega}\right\rangle .
\end{aligned}
$$

We prove the following.

Proposition 14. Let $\gamma=\kappa_{1}<\kappa_{2}$. Then, there exists a constant $\varepsilon_{0}>0$ such that

$$
E\left(\vec{\phi}_{\omega}\right) \leq E(\vec{u})-\frac{\left(M(\vec{u}), \vec{\psi}_{\omega}\right)_{H}}{2\left\|\vec{\psi}_{\omega}\right\|_{H}^{2}} P(\vec{u})
$$

for all $\vec{u} \in U_{\varepsilon_{0}}\left(\vec{\phi}_{\omega}\right)$ satisfying $Q(\vec{u})=Q\left(\vec{\phi}_{\omega}\right)$.

For the proof of Proposition 14, we prove several lemmas.

Lemma 15. For $\lambda \in \mathbb{R}$ and $\vec{z} \in X$,

$$
\begin{aligned}
& \lambda\left\langle S_{\omega}^{\prime}\left(\vec{\phi}_{\omega}+\lambda \vec{\psi}_{\omega}+\vec{z}\right), \vec{\psi}_{\omega}\right\rangle \\
& =\frac{\nu_{1}}{3 !} \lambda^{4}+\lambda^{2}\left\langle S_{\omega}^{(3)}\left(\vec{\phi}_{\omega}\right)\left(\vec{\psi}_{\omega}, \vec{\psi}_{\omega}\right), \vec{z}\right\rangle+o\left(\lambda^{4}+\|\vec{z}\|_{X}^{2}\right) .
\end{aligned}
$$


Proof. By Taylor's expansion, we have

$$
\begin{aligned}
& \lambda\left\langle S_{\omega}^{\prime}\left(\vec{\phi}_{\omega}+\lambda \vec{\psi}_{\omega}+\vec{z}\right), \vec{\psi}_{\omega}\right\rangle \\
& =\lambda\left\langle S_{\omega}^{\prime}\left(\vec{\phi}_{\omega}+\lambda \vec{\psi}_{\omega}\right), \vec{\psi}_{\omega}\right\rangle+\lambda\left\langle S_{\omega}^{\prime \prime}\left(\vec{\phi}_{\omega}+\lambda \vec{\psi}_{\omega}\right) \vec{z}, \vec{\psi}_{\omega}\right\rangle+O\left(\lambda\|\vec{z}\|_{X}^{2}\right) .
\end{aligned}
$$

Here, by Lemma 5, we have $\lambda\left\langle S_{\omega}^{\prime}\left(\vec{\phi}_{\omega}+\lambda \vec{\psi}_{\omega}\right), \vec{\psi}_{\omega}\right\rangle=\frac{\nu_{1}}{3 !} \lambda^{4}$.

Next, since $S_{\omega}^{\prime \prime}\left(\vec{\phi}_{\omega}\right) \vec{\psi}_{\omega}=0$, we have

$$
\begin{aligned}
& \lambda\left\langle S_{\omega}^{\prime \prime}\left(\vec{\phi}_{\omega}+\lambda \vec{\psi}_{\omega}\right) \vec{z}_{,} \vec{\psi}_{\omega}\right\rangle \\
& =\lambda\left\langle S_{\omega}^{\prime \prime}\left(\vec{\phi}_{\omega}\right) \vec{z}, \vec{\psi}_{\omega}\right\rangle+\lambda^{2}\left\langle S_{\omega}^{(3)}\left(\vec{\phi}_{\omega}\right)\left(\vec{\psi}_{\omega}, \vec{z}\right), \vec{\psi}_{\omega}\right\rangle+o\left(\lambda^{2}\|\vec{z}\|_{X}\right) \\
& =\lambda^{2}\left\langle S_{\omega}^{(3)}\left(\vec{\phi}_{\omega}\right)\left(\vec{\psi}_{\omega}, \vec{\psi}_{\omega}\right), \vec{z}\right\rangle+o\left(\lambda^{2}\|\vec{z}\|_{X}\right) .
\end{aligned}
$$

Thus, we obtain the desired result.

Lemma 16. For $\lambda \in \mathbb{R}$ and $\vec{z} \in X$,

$$
\begin{aligned}
& \lambda^{2}\left\langle S_{\omega}^{\prime}\left(\vec{\phi}_{\omega}+\lambda \vec{\psi}_{\omega}+\vec{z}\right), \vec{\phi}_{\omega}\right\rangle \\
& =\frac{\lambda^{4}}{2}\left\langle S_{\omega}^{(3)}\left(\vec{\phi}_{\omega}\right)\left(\vec{\psi}_{\omega}, \vec{\psi}_{\omega}\right), \vec{\phi}_{\omega}\right\rangle+\lambda^{2}\left\langle S_{\omega}^{\prime \prime}\left(\vec{\phi}_{\omega}\right) \vec{\phi}_{\omega}, \vec{z}\right\rangle+o\left(\lambda^{4}+\|\vec{z}\|_{X}^{2}\right) .
\end{aligned}
$$

Proof. By Taylor's expansion, we have

$$
\begin{aligned}
& \left\langle S_{\omega}^{\prime}\left(\vec{\phi}_{\omega}+\lambda \vec{\psi}_{\omega}+\vec{z}\right), \vec{\phi}_{\omega}\right\rangle \\
& =\left\langle S_{\omega}^{\prime}\left(\vec{\phi}_{\omega}+\lambda \vec{\psi}_{\omega}\right), \vec{\phi}_{\omega}\right\rangle+\left\langle S_{\omega}^{\prime \prime}\left(\vec{\phi}_{\omega}+\lambda \vec{\psi}_{\omega}\right) \vec{z}, \vec{\phi}_{\omega}\right\rangle+O\left(\|\vec{z}\|_{X}^{2}\right) .
\end{aligned}
$$

Here, it follows from Lemma 6 that

$$
\left\langle S_{\omega}^{\prime}\left(\vec{\phi}_{\omega}+\lambda \vec{\psi}_{\omega}\right), \vec{\phi}_{\omega}\right\rangle=\frac{\lambda^{2}}{2}\left\langle S_{\omega}^{(3)}\left(\vec{\phi}_{\omega}\right)\left(\vec{\psi}_{\omega}, \vec{\psi}_{\omega}\right), \vec{\phi}_{\omega}\right\rangle+o\left(\lambda^{2}\right)
$$

Moreover, we have $\left\langle S_{\omega}^{\prime \prime}\left(\vec{\phi}_{\omega}+\lambda \vec{\psi}_{\omega}\right) \vec{z}, \vec{\phi}_{\omega}\right\rangle=\left\langle S_{\omega}^{\prime \prime}\left(\vec{\phi}_{\omega}\right) \vec{z}, \vec{\phi}_{\omega}\right\rangle+O\left(\lambda\|\vec{z}\|_{X}\right)$.

Thus, we have

$$
\begin{aligned}
\left\langle S_{\omega}^{\prime}\left(\vec{\phi}_{\omega}+\lambda \vec{\psi}_{\omega}+\vec{z}\right), \vec{\phi}_{\omega}\right\rangle=\frac{\lambda^{2}}{2}\left\langle S_{\omega}^{(3)}\left(\vec{\phi}_{\omega}\right)\left(\vec{\psi}_{\omega}, \vec{\psi}_{\omega}\right), \vec{\phi}_{\omega}\right\rangle \\
\quad+\left\langle S_{\omega}^{\prime \prime}\left(\vec{\phi}_{\omega}\right) \vec{\phi}_{\omega}, \vec{z}\right\rangle+o\left(\lambda^{2}\right)+O\left(\lambda\|\vec{z}\|_{X}\right)+O\left(\|\vec{z}\|_{X}^{2}\right),
\end{aligned}
$$

which implies the desired result.

Lemma 17. Let $\vec{v}=\lambda \vec{\psi}_{\omega}+\mu \vec{\phi}_{\omega}+\vec{w}$ with $\lambda, \mu \in \mathbb{R}$ and $\vec{w} \in W$. Assume that $\left\|\vec{\phi}_{\omega}+\vec{v}\right\|_{H}^{2}=\left\|\vec{\phi}_{\omega}\right\|_{H}^{2}$. Then,

$$
\lambda\left\langle S_{\omega}^{\prime}\left(\vec{\phi}_{\omega}+\vec{v}\right), \vec{\psi}_{\omega}\right\rangle-\lambda^{2}\left\langle S_{\omega}^{\prime}\left(\vec{\phi}_{\omega}+\vec{v}\right), \vec{\phi}_{\omega}\right\rangle=4 \nu_{0} \lambda^{4}+o\left(\lambda^{4}+\|\vec{w}\|_{X}^{2}\right) .
$$


Proof. By Lemmas 8 and [15, we have

$$
\begin{aligned}
& \lambda\left\langle S_{\omega}^{\prime}\left(\vec{\phi}_{\omega}+\vec{v}\right), \vec{\psi}_{\omega}\right\rangle=\lambda\left\langle S_{\omega}^{\prime}\left(\vec{\phi}_{\omega}+\lambda \vec{\psi}_{\omega}+\mu \vec{\phi}_{\omega}+\vec{w}\right), \vec{\psi}_{\omega}\right\rangle \\
& =\frac{\nu_{1}}{3 !} \lambda^{4}-\frac{\lambda^{4}}{2}\left\langle S_{\omega}^{(3)}\left(\vec{\phi}_{\omega}\right)\left(\vec{\psi}_{\omega}, \vec{\psi}_{\omega}\right), \vec{\phi}_{\omega}\right\rangle \\
& \quad+\lambda^{2}\left\langle S_{\omega}^{(3)}\left(\vec{\phi}_{\omega}\right)\left(\vec{\psi}_{\omega}, \vec{\psi}_{\omega}\right), \vec{w}\right\rangle+o\left(\lambda^{4}+\|\vec{w}\|_{X}^{2}\right) .
\end{aligned}
$$

On the other hand, by Lemmas 8 and [16, we have

$$
\begin{gathered}
\lambda^{2}\left\langle S_{\omega}^{\prime}\left(\vec{\phi}_{\omega}+\vec{v}\right), \vec{\phi}_{\omega}\right\rangle=\lambda^{2}\left\langle S_{\omega}^{\prime}\left(\vec{\phi}_{\omega}+\lambda \vec{\psi}_{\omega}+\mu \vec{\phi}_{\omega}+\vec{w}\right), \vec{\phi}_{\omega}\right\rangle \\
=\frac{\lambda^{4}}{2}\left\langle S_{\omega}^{(3)}\left(\vec{\phi}_{\omega}\right)\left(\vec{\psi}_{\omega}, \vec{\psi}_{\omega}\right), \vec{\phi}_{\omega}\right\rangle+\lambda^{2} \mu\left\langle S_{\omega}^{\prime \prime}\left(\vec{\phi}_{\omega}\right) \vec{\phi}_{\omega}, \vec{\phi}_{\omega}\right\rangle \\
\quad+\lambda^{2}\left\langle S_{\omega}^{\prime \prime}\left(\vec{\phi}_{\omega}\right) \vec{\phi}_{\omega}, \vec{w}\right\rangle+o\left(\lambda^{4}+\left\|\mu \vec{\phi}_{\omega}+\vec{w}\right\|_{X}^{2}\right) \\
=\frac{\lambda^{4}}{2}\left\langle S_{\omega}^{(3)}\left(\vec{\phi}_{\omega}\right)\left(\vec{\psi}_{\omega}, \vec{\psi}_{\omega}\right), \vec{\phi}_{\omega}\right\rangle-\frac{\lambda^{4}}{2}\left\langle S_{\omega}^{\prime \prime}\left(\vec{\phi}_{\omega}\right) \vec{\phi}_{\omega}, \vec{\phi}_{\omega}\right\rangle \\
\quad+\lambda^{2}\left\langle S_{\omega}^{\prime \prime}\left(\vec{\phi}_{\omega}\right) \vec{\phi}_{\omega}, \vec{w}\right\rangle+o\left(\lambda^{4}+\|\vec{w}\|_{X}^{2}\right) .
\end{gathered}
$$

Thus, we have

$$
\begin{aligned}
\lambda\left\langle S_{\omega}^{\prime}\left(\vec{\phi}_{\omega}+\vec{v}\right), \vec{\psi}_{\omega}\right\rangle-\lambda^{2}\left\langle S_{\omega}^{\prime}\left(\vec{\phi}_{\omega}+\vec{v}\right), \vec{\phi}_{\omega}\right\rangle \\
=4 \nu_{0} \lambda^{4}-\lambda^{2}\left\langle S_{\omega}^{\prime \prime}\left(\vec{\phi}_{\omega}\right) \vec{\phi}_{\omega}, \vec{w}\right\rangle+\lambda^{2}\left\langle S_{\omega}^{(3)}\left(\vec{\phi}_{\omega}\right)\left(\vec{\psi}_{\omega}, \vec{\psi}_{\omega}\right), \vec{w}\right\rangle \\
\quad+o\left(\lambda^{4}+\|\vec{w}\|_{X}^{2}\right) .
\end{aligned}
$$

Finally, by (9), we obtain the desired result.

We are now in a position to give the proof of Proposition 14,

Proof of Proposition 14. Let $\vec{u} \in U_{\varepsilon}\left(\vec{\phi}_{\omega}\right)$ with $Q(\vec{u})=Q\left(\vec{\phi}_{\omega}\right)$. We put $\vec{v}=$ $M(\vec{u})-\vec{\phi}_{\omega}$, and decompose $\vec{v}$ as $\vec{v}=\lambda \vec{\psi}_{\omega}+\mu \vec{\phi}_{\omega}+\vec{w}$ with $\lambda, \mu \in \mathbb{R}$ and $\vec{w} \in W$. Here, we note that $\left(\vec{v}, i \vec{\phi}_{\omega}\right)_{H}=0$ by Lemma 11,

$$
\lambda=\frac{\left(\vec{v}, \vec{\psi}_{\omega}\right)_{H}}{\left\|\vec{\psi}_{\omega}\right\|_{H}^{2}}=\frac{\left(M(\vec{u}), \vec{\psi}_{\omega}\right)_{H}}{\left\|\vec{\psi}_{\omega}\right\|_{H}^{2}},
$$

and $\|\vec{v}\|_{X} \leq C \varepsilon$ for some $C>0$ by (12).

Since $\left\|\vec{\phi}_{\omega}+\vec{v}\right\|_{H}^{2}=\|\vec{u}\|_{H}^{2}=2 Q(\vec{u})=2 Q\left(\vec{\phi}_{\omega}\right)=\left\|\vec{\phi}_{\omega}\right\|_{H}^{2}$, it follows from Lemmas 9 and 4 that

$$
E(\vec{u})-E\left(\vec{\phi}_{\omega}\right) \geq \nu_{0} \lambda^{4}+\frac{k_{0}}{2}\|\vec{w}\|_{X}^{2}+o\left(\lambda^{4}+\|\vec{w}\|_{X}^{2}\right) .
$$


Moreover, by (14) and Lemmas 8, 16 and 17, we have

$$
\begin{aligned}
& \lambda P(\vec{u})=\lambda\left\langle S_{\omega}^{\prime}\left(\vec{\phi}_{\omega}+\vec{v}\right), \vec{\psi}_{\omega}\right\rangle-\lambda \frac{\left(\vec{\phi}_{\omega}+\vec{v}, \vec{\psi}_{\omega}\right)_{H}}{\left(\vec{\phi}_{\omega}+\vec{v}, \vec{\phi}_{\omega}\right)_{H}}\left\langle S_{\omega}^{\prime}\left(\vec{\phi}_{\omega}+\vec{v}\right), \vec{\phi}_{\omega}\right\rangle \\
& =\lambda\left\langle S_{\omega}^{\prime}\left(\vec{\phi}_{\omega}+\vec{v}\right), \vec{\psi}_{\omega}\right\rangle-\frac{\lambda^{2}\left\|\vec{\psi}_{\omega}\right\|_{H}^{2}}{(1+\mu)\left\|\vec{\phi}_{\omega}\right\|_{H}^{2}}\left\langle S_{\omega}^{\prime}\left(\vec{\phi}_{\omega}+\vec{v}\right), \vec{\phi}_{\omega}\right\rangle \\
& =\lambda\left\langle S_{\omega}^{\prime}\left(\vec{\psi}_{\omega}+\vec{v}\right), \vec{\psi}_{\omega}\right\rangle-\lambda^{2}\left\langle S_{\omega}^{\prime}\left(\vec{\phi}_{\omega}+\vec{v}\right), \vec{\phi}_{\omega}\right\rangle+o\left(\lambda^{4}+\|\vec{w}\|_{X}^{2}\right) \\
& =4 \nu_{0} \lambda^{4}+o\left(\lambda^{4}+\|\vec{w}\|_{X}^{2}\right) .
\end{aligned}
$$

Here, we used the fact that $\left\|\vec{\psi}_{\omega}\right\|_{H}=\left\|\vec{\phi}_{\omega}\right\|_{H}$. Thus, we have

$$
E(\vec{u})-E\left(\vec{\phi}_{\omega}\right)-\frac{\lambda}{2} P(\vec{u}) \geq-\nu_{0} \lambda^{4}+\frac{k_{0}}{2}\|\vec{w}\|_{X}^{2}+o\left(\lambda^{4}+\|\vec{w}\|_{X}^{2}\right) .
$$

Since $k_{0}>0$ and $-\nu_{0}>0$ by (10) and the assumption $\kappa_{1}<\kappa_{2}$, taking $\varepsilon$ smaller if necessary, we have

$$
E(\vec{u})-E\left(\vec{\phi}_{\omega}\right) \geq \frac{\lambda}{2} P(\vec{u})=\frac{\left(M(\vec{u}), \vec{\psi}_{\omega}\right)_{H}}{2\left\|\vec{\psi}_{\omega}\right\|_{H}^{2}} P(\vec{u}) .
$$

This completes the proof.

Finally, we prove the instability part of Theorem 2 .

Proof of Theorem 2 (Instability part). Suppose that $e^{i \omega t} \vec{\phi}_{\omega}$ is stable. For $\lambda$ close to 0 , we define

$$
\vec{\varphi}_{\lambda}=\vec{\phi}_{\omega}+\lambda \vec{\psi}_{\omega}+\sigma(\lambda) \vec{\phi}_{\omega}, \quad \sigma(\lambda)=\left(1-\lambda^{2}\right)^{1 / 2}-1 .
$$

Then, we have $Q\left(\vec{\varphi}_{\lambda}\right)=Q\left(\vec{\phi}_{\omega}\right)$. Moreover, since $\nu_{0}<0$, by Lemma 9, there exists $\lambda_{1}>0$ such that

$$
\delta_{\lambda}:=E\left(\vec{\phi}_{\omega}\right)-E\left(\vec{\varphi}_{\lambda}\right)=-\nu_{0} \lambda^{4}+o\left(\lambda^{4}\right)>0
$$

for $\lambda \in\left(-\lambda_{1}, 0\right) \cup\left(0, \lambda_{1}\right)$.

Let $\vec{u}_{\lambda}(t)$ be the solution of (11) with $\vec{u}_{\lambda}(0)=\vec{\varphi}_{\lambda}$. Since $e^{i \omega t} \vec{\phi}_{\omega}$ is stable, there exists $\lambda_{0} \in\left(0, \lambda_{1}\right)$ such that if $|\lambda|<\lambda_{0}$, then $\vec{u}_{\lambda}(t) \in U_{\varepsilon_{0}}\left(\vec{\phi}_{\omega}\right)$ for all $t \geq 0$, where $\varepsilon_{0}$ is the positive conatant given in Proposition 14 .

By the definition (13) of $M$ and $A$, there exist positive constants $C_{1}$ and $C_{2}$ such that

$$
|M(\vec{v})| \leq C_{1}\left\|\vec{\psi}_{\omega}\right\|_{H}, \quad|A(\vec{v})| \leq C_{2}
$$

for all $\vec{v} \in U_{\varepsilon_{0}}\left(\vec{\phi}_{\omega}\right)$. 
For $\lambda \in\left(-\lambda_{0}, 0\right) \cup\left(0, \lambda_{0}\right)$, by Proposition 14 and the conservation of $E$ and $Q$, we have

$$
0<\delta_{\lambda}=E\left(\vec{\phi}_{\omega}\right)-E\left(\vec{u}_{\lambda}(t)\right) \leq C_{1}\left|P\left(\vec{u}_{\lambda}(t)\right)\right|
$$

for all $t \geq 0$. Since $t \mapsto P\left(\vec{u}_{\lambda}(t)\right)$ is continuous, we see that either (i) $P\left(\vec{u}_{\lambda}(t)\right) \geq \delta_{\lambda} / C_{1}$ for all $t \geq 0$, or (ii) $P\left(\vec{u}_{\lambda}(t)\right) \leq-\delta_{\lambda} / C_{1}$ for all $t \geq 0$. Moreover, by Lemma 13, we have

$$
\frac{d}{d t} A\left(\vec{u}_{\lambda}(t)\right)=P\left(\vec{u}_{\lambda}(t)\right)
$$

for all $t \geq 0$. Therefore, we see that $\left|A\left(\vec{u}_{\lambda}(t)\right)\right| \rightarrow \infty$ as $t \rightarrow \infty$. This contradicts the fact that $\left|A\left(\vec{u}_{\lambda}(t)\right)\right| \leq C_{2}$ for all $t \geq 0$. Hence, $e^{i \omega t} \vec{\phi}_{\omega}$ is unstable.

Acknowledgment. This work was supported by JSPS KAKENHI Grant Numbers 15K04968, 26247013.

\section{References}

[1] G. Agrawal, Nonlinear fiber optics, Optics and Photonics, Academic Press, 2007.

[2] T. Cazenave, Semilinear Schrödinger equations, Courant Lecture Notes in Mathematics 10, Amer. Math. Soc., Providende, RI, 2003.

[3] M. Colin, T. Colin and M. Ohta, Stability of solitary waves for a system of nonlinear Schrödinger equations with three wave interaction, Ann. Inst. H. Poincaré, Anal. Non Linéaire, 26 (2009), 2211-2226.

[4] M. Colin, T. Colin and M. Ohta, Instability of standing waves for a system of nonlinear Schrödinger equations with three-wave interaction, Funkcial. Ekvac. 52 (2009), 371-380.

[5] M. Colin and M. Ohta, Bifurcation from semitrivial standing waves and ground states for a system of nonlinear Schrödinger equations, SIAM J. Math. Anal. 44 (2012), 206-223.

[6] M. Grillakis, J. Shatah and W. Strauss, Stability theory of solitary waves in the presence of symmetry, I, J. Funct. Anal., 74 (1987), 160-197.

[7] N. Hayashi, T. Ozawa and K. Tanaka, On a system of nonlinear Schrödinger equations with quadratic interaction, Ann. Inst. H. Poincaré, Anal. Non Linéaire, 30 (2013), 661-690. 
[8] J. Ieda, T. Miyakawa and M. Wadati, Matter-wave solitons in an $F=1$ spinor Bose-Einstein condensate, J. Phys. Soc. Jpn., 73 (2004), 29963007 .

[9] T. Kanna and K. Sakkaravarthi, Multicomponent coherently coupled and incoherently coupled solitons and their collisions, J. Phys. A: Math. Theor., 44 (2011), 285211 (30pp).

[10] M. Maeda, Stability of bound states of Hamiltonian PDEs in the degenerate cases, J. Funct. Anal., 263 (2012), 511-528.

[11] N. Nguyen and Z.-Q. Wang, Orbital stability of solitary waves for a nonlinear Schrödinger system, Adv. Differential Equations, 16 (2011), $977-1000$.

[12] M. Ohta, Stability of solitary waves for coupled nonlinear Schrödinger equations, Nonlinear Anal., 26 (1996), 933-939.

[13] M. Ohta, Instability of bound states for abstract nonlinear Schrödinger equations, J. Funct. Anal., 261 (2011), 90-110.

[14] Z. Wang and S. Cui, Multi-speed solitary wave solutions for a coherently coupled nonlinear Schrödinger system, J. Math. Phys., 56 (2015), no. 2, 021503, 13 pp.

[15] M. I. Weinstein, Modulational stability of ground states of nonlinear Schrödinger equations, SIAM J. Math. Anal., 16 (1985), 472-491.

[16] M. I. Weinstein, Lyapunov stability of ground states of nonlinear dispersive evolution equations, Comm. Pure Appl. Math., 39 (1986), 51-68.

[17] Y. Yamazaki, Stability of line standing waves near the bifurcation point for nonlinear Schrödinger equations, Kodai Math. J., 38 (2015), 65-96. 